\title{
Diptères Tachinaires de Richelieu (Indre-et-Loire)
}

\author{
Par Jehoshua KUGLER
}

Grâce à la bienveillante invitation de M. C. Dupuis, Sous-Directeur de la Station de Parasitologie expérimentale et comparée de Richelieu, j'ai eu l'occasion de pouvoir étudier durant deux été consécutifs, du 20 août au 14 septembre 1964 , et du $1^{\text {er }}$ août au 14 septembre 1965, les Diptères Tachinaires du Domaine de l'Université de Paris à Richelieu (Indre-et-Loire). J'ai pu, de la sorte, me familiariser avec la faune tachinologique ouest-européenne, qui diffère beaucoup de celle d'Israël puisque sur les 102 espèces de Tachinaires qu'il m'a été donné d'observer à Richelieu, 30 seulement existent en Israël (Kugler, 1963).

Le Domaine de Richelieu est situé au sud-ouest du département d'Indre-et-Loire, près de la ville de Richelieu; il s'étend sur 500 hectares, dont 300 ha de forêt et 200 de parc et de cultures; les grandes lignes de la physiographie locale ont été précisées par Dupuis (1963a).

Il convient de noter que les conditions météorologiques locales peuvent varier considérablement selon les années et que tel fut précisément le cas pour les étés 1964 et 1965 , radicalement différents l'un de l'autre. L'été, 1964, avait été marqué par une sécheresse et une chaleur au-dessus de la normale ; à mon arrivée, le 20 août, les arbres portaient déjà leur parure automnale. L'été de 1965 fut, par contre, pluvieux et froid et certains jours de septembre la température, entre 6 et $8 \mathrm{~h}$ du matin, est tombée jusqu'à $5^{\circ} \mathrm{C}$. Ces différences de température ont, sans nul doute, affecté les Tachinaires, car une partie des espèces récoltées au début de mon séjour en 1964 n'ont pu être capturées, en 1965, que vers la fin de l'été.

Nous avons procédé à deux types de récoltes.

Les récoltes du premier type étaient effectuées entre $10 \mathrm{~h} 30$ et $13 \mathrm{~h}$ sur les fleurs, en particulier de Pastinaca sativa L. et de Daucus carota L., qui croissent le long des chemins et entre les arbres des jeunes plantations de peupliers; dans ces mêmes conditions, des mouches ont été récoltées sur les fleurs suivantes: Heracleum sphondylium L., Leucanthemum vulgare Lmk., Achillea millefolium L., Eryngium campestre L., Erigeron canadensis L., Torilis anthriscus L. et Ormenis mixta L.

Les récoltes du second type avaient lieu au coucher du soleil, c'est-à-dire, pour les dates considérées, entre $18 \mathrm{~h}$ et $20 \mathrm{~h}$ (heure légale française), sur des végétaux secs, en particulier sur Crepis sp., Senecio jacobaea L. et différentes Gramineae. Les inté- 
ressants résultats de ces « chasses au couchant», qui nous furent enseignées par M. Cl. Dupuis, indiquent que beaucoup de Tachinaires passent la nuit, comme les Phasiinae, sur les plantes sèches.

Mes déterminations, effectuées à la Station de Richelieu, à l'aide de la littérature se trouvant sur place, ont été précisées au Muséum national d'Histoire naturelle à Paris et au Commonwealth Institute of Biological Control, European Station, Delémont (Suisse).

La liste que nous donnons ci-après comprend 71 espèces de Tachinaires (toutes de nos récoltes personnelles, à l'exception des Freraea et de trois Strongygastrini récoltés antérieurement par C. Dupuis). Nous n'y avons pas fait figurer 31 espèces de Phasiinae, sous-famille qui fait l'objet des recherches du $\mathrm{D}^{\mathrm{r}}$ Dupuis depuis de nombreuses années, car les résultats qui la concernent ont été publiés par cet auteur dans diverses contributions ou seront complétés dans ses contributions ultérieures.

Notre liste mentionne les dates et plantes de récoltes de nos spécimens de Richelieu et rappelle pour chaque espèce l'identité de ses hôtes et sa distribution générale. Des listes complètes d'hôtes figurent dans les publications de Herting (1960) et Mesnil (1944-1966). Nous ne mentionnons pas de synonymies, cependant, lorsque certaines espèces figurent sous des noms différents dans d'importants travaux modernes, nous mentionnons ces noms entre parenthèses en indiquant par une lettre majuscule l'auteur du travail considéré : $\mathrm{S}=$ Stein, $\mathrm{L}=$ Lundbeck, $\mathrm{M}=$ Mesnil, $\mathrm{E}=$ van Emden et $\mathbf{H}=$ Herting.

L'auteur tient à exprimer sa profonde reconnaissance au $\mathrm{D}^{\mathrm{r}} \mathrm{C}$. Dupuis pour son aimable hospitalité et son assistance durant ses séjours à la Station de Richelieu et au Muséum National d'Histoire Naturelle à Paris, ainsi qu'aux $\mathrm{D}^{\mathrm{rs}}$ L.-P. Mesnil et B. Herting pour leur hospitalité au Commonwealth Institute à Delémont. II remercie également le Centre National de la Recherche Scientifique (France) qui, dans le cadre de la convention d'échange de travailleurs scientifiques entre la France et Israël, lui a permis son séjour en France en 1965.

\section{Liste des Espèces}

\section{EXORISTINI}

1) Exorista larvarum (L.) (Tachina, S, L, E). - 1 ơ, 21-VIII-64 ; 1 q, 31-VIII-

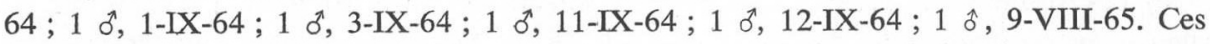
mouches ont été récoltées sur Pastinaca et Daucus.

Hôtes: Herting (1960) donne une liste de plus de 50 espèces différentes de Chenilles de Lépidoptères, hôtes de ce Diptère.

Distribution: Abondante en Europe centrale; atteint l'Ecosse et la Laponie ; Sibérie jusqu'à la région de l'Amour ; se récolte aussi en Israël ; introduite aux U.S.A.

2) Exorista fasciata (Fall.) (Tachina, S, E, L). - 1 d, 9-VIII-65 sur Pastinaca; $1 \delta$, 20-VIII-65 avant le coucher du soleil sur Crepis sec. 
Hôtes : Chenilles de divers Lépidoptères.

Distribution : Europe.

3) Exorista rustica (Fall.) (Tachina, S, L, E). - Cette Tachinaire est l'une des plus communes à Richelieu; nous l'avons récoltée en nombre, tant en 1964 qu'en 1965, chaque jour sur les Pastinaca et Daucus et également au couchant sur les Crepis. Le 12-VIII-65, nous avons noté un couple in copula sur Crepis sec.

Hôtes : Larves des Tenthredinidae.

Distribution: Europe ; atteint la Suède, la Finlande, le Nord de l'Angleterre ; Asie centrale (Kazakhstan) ; Vladivostok ; se trouve également en Israël.

4) Exorista pratensis (R. D.) (erucarum Rond., H, Tachina erucarum Rond., E).

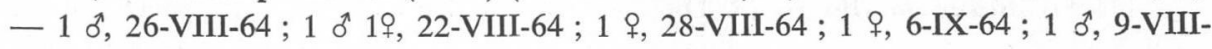
65, sur Pastinaca.

HôTEs: Larves de Tenthredinidae.

Distribution : Europe, Israël.

5) Meigenia mutabilis (Fall.). - 1 d, 5-IX-64; 1 б, 6-IX-64; 2 \%, 10-IX-64 ; 2 o, 11-IX-64; 1 ㅇ, 29-VIII-65; 1 o, 30-VIII-65; 1 ㅇ, 1-IX-65; 1 o, 9-IX-65, sur Pastinaca.

Hôtes. - Larves de différentes espèces de Chrysomelidae; élevée également de fausses chenilles de Tenthredinidae du genre Athalia.

Distribution: Europe ; atteint l'Ecosse et le Nord de la Scandinavie ; Asie centrale (Tadjikistan); Israël.

6) Perichateta unicolor (Fall.). - 1 \%, 8-IX-64; 2 q, 10-IX-64; 1 +, 11-IX-64; 1 ô, 12-IX-64, récoltés sur des Pastinaca.

Hôtes: Larves de Chrysomelidae.

Distribution: Europe centrale, Angleterre, Finlande, environs de Léningrad, Ukraine.

7) Arrhinomyia innoxia (Meig.) (Apatelia S). - 1 , récoltée sur Pastinaca le 24-VIII-64.

Hôtes: Les hôtes de cette espèce ne sont pas connus avec certitude; d'après Mesnil, on peut supposer que ce sont des Coléoptères.

Distribution: Espèce rare; Europe jusqu'au Sud de l'Angleterre et en Finlande.

8) Anachetopsis angustifrons (Loew) (ocypterina Zett., S, L ; zetterstedti Ringd., E; Ligeria angusticornis Loew, M). - C'est l'une des espèces les plus abondantes de Richelieu; nous l'avons capturée en quantité, tant en 1964 qu'en 1965, pendant le jour sur les Pastinaca et au couchant sur les Crepis secs.

Hôtes: Larves de Pterophoridae.

Distribution: Europe centrale, environs de Léningrad, Angleterre ; non signalé de Scandinavie.

9) Compsilura concinnata (Meig.). - 1 o, 24-VIII-64; 1 ㅇ, 1-IX-64; 1 t, 2-IX-64; 2 o, 11-IX-64, tous sur Pastinaca.

Hôtes: Cette espèce, très polyphage, compte plus de 200 hôtes différents; ce 
sont, le plus souvent, des chenilles de Macrolépidoptères de diverses familles et, plus rarement, des larves de Tenthredinidae.

Distribution: Abondante en Europe jusqu'au Nord de l'Allemagne et dans le Sud de l'Angleterre; d'après Mesnil, elle est également commune en Asie jusqu'au Japon; nous l'avons trouvée en Israël; elle a été introduite en Amérique du Nord.

10) Acemyia acuticornis (Meig.). - 1 ơ, 1-IX-64, sur Daucus.

Hôtes. - Espèces diverses d'Acrididae, tant à Richelieu (Callot 1937) qu'en Israël (Tmethis pulchripennis asiaticus Uv., cf. Shulov 1952).

Distribution: Europe centrale et méridionale; atteint la Finlande et la Suède centrale et est abondante aux environs de Léningrad; Israël.

11) Strobliomyia tibialis (R. D.) (Actia S). - De nombreux spécimens ont été récoltés pendant le jour sur les Pastinaca et au couchant sur les Crepis secs ; c'est l'une des espèces les plus courantes à Richelieu.

Hôtes: Chenilles de différents Lépidoptères. En Israël, nous l'avons élevée, surtout en hiver, sur des chenilles d'Orgyia dubia Tausch (Orgyidae) et de Lasiocampa grandis Stgr. (Lasiocampidae) (Kugler 1961).

Distribution: D'après Mesnil, l'espèce est largement répandue dans la région paléarctique, y compris le Japon ; en Europe, elle atteint le Nord de l'Allemagne ; on ne l'a signalée ni d'Angleterre ni de Scandinavie. Elle existe en Afrique et en Israël.

12) Actia pilipennis (Fall.). - 1 \%, 3-IX-64, sur Senecio jacobaea sec, le soir ; 1 o, 9-IX-65, sur Pastinaca durant le jour.

Hôtes: A. pilipennis est connue comme parasite des larves de diverses espèces, principalement de Tortricidae, mais aussi de Pterophoridae.

Distribution : Europe jusqu'au Nord de la Scandinavie.

13) Actia crassicornis (Meig.). - Récoltée sur des Senecio et des Graminées

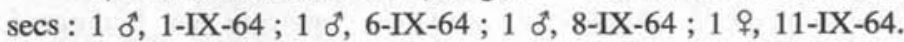

Hôtes: Chenilles de Gelechiidae et de Tortricidae.

Distribution: Europe.

14) Actia infantula (Zett.) (nigroscutellata Vill., L, antennalis Rond., E). -

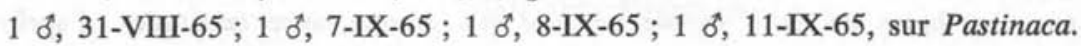

Hôtes : Larves de Tineidae.

Distribution: Surtout dans les régions chaudes d'Europe, jusqu'au Sud de l'Angleterre et en Suède centrale.

15) Siphona geniculata (Deg.). - 1 శ, 8-IX-65, sur Pastinaca.

Hôtes: Larves de Tipulidae.

Distribution : Europe.

16) Siphona delicatula Mesn. - 1 ఠ, 3-IX-65; 1 ఠ, 7-IX-65; 1 \&, 11-IX-65, capturés entre 17 et 19 h, sur les Pastinaca.

Hôtes : Inconnus.

Distribution: Décrite par Mesnil (1960) d'après des exemplaires récoltés en Ecosse, l'espèce a été récoltée depuis en Autriche et aux environs de Léningrad. Nous la mentionnons de France pour la première fois. 


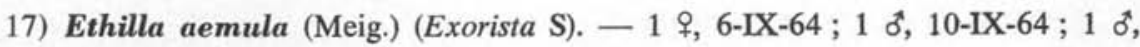
12-VIII-65, récoltés sur Pastinaca.

Hôtes : Inconnus.

Distribution: Régions chaudes d'Europe; atteint l'Autriche.

\section{GONIINI}

18) Aplomyia confinis (Fall.) (Exorista S, L, Zenillia, E). - C'est l'une des Tachinaires les plus communes de Richelieu; nous l'avons prise de jour sur les Pastinaca et Daucus et au couchant sur les Crepis secs.

Hôtes : Diverses espèces de Lycaenidae.

Distribution: Régions chaudes d'Europe, jusqu'en Angleterre méridionale et en Suède centrale ; Asie centrale (Kazakhstan) ; Vladivostok; Afrique du Nord, Israël.

19) Epicampocera succincta (Meig.). - Cette espèce était très abondante en 1964 et de nombreux spécimens ont été récoltés entre le 20 août et le 11 septembre ; elle était par contre très rare en $1965 ; 2 \sigma^{\star}$ et 2 o seulement du 7 au 12 septembre.

Hôtes: Chenilles de Pieris rapae L., Pieris napi L., (Pieridae) et de Boarmia selenaria Schiff. (Geometridae).

Distribution : Europe, sauf la Scandinavie.

20) Phryxe vulgaris (Fall.) (Zenillia E). - C'est l'une des espèces les plus abondantes à Richelieu; nous avons récolté de nombreux spécimens en 1964 et 1965 sur des Pastinaca et Daucus.

Hôtes : Ph. vulgaris est un polyphage, parasite des différentes espèces de Macrolépidoptères; en Israël, elle a été élevée de Pieris brassicae L. et Euchloe orientalis mellisanda Fruhst.

Distribution : abondante en Europe et en Israël ; Sibérie jusqu'à la région de Vladivostok.

21) Phryxe nemea (Meig.) (Zenillia E). - 1 f, 14-VIII-65, sur Pastinaca.

HôTEs: Nombreuses espèces de Macrolépidoptères et aussi quelques espèces de Microlépidoptères.

DisTRIBUTION : Europe.

22) Catagonia aberrans (Rond.) (Exorista S, Sisyropa M). -1 \&, 19-VIII-65, sur Pastinaca.

Hôtes : Inconnus.

Distribution: Europe méridionale ; atteint l'Allemagne.

23) Chaetina setigena (Rond.) (Chaetolya S). - 1 d, 20-VIII-65, sur Eryngium campestre.

HôTES : Inconnus.

Distribution : Espèce rare, connue de Corse, du Midi de la France, du Liban et d'Israël.

24) Lydella stabulans (Meig.) (Ceromasia S, Paraphorocera E). - L'une des espèces les plus communes de Richelieu; nombreux spécimens récoltés sur Pastinaca; moins fréquente sur Crepis sec. 
Hôtes: Chenilles de Noctuidae.

Distribution : Europe.

25) Lydella grisescens R. D. (Ceromasia S, Paraphorocera senilis Meig. E). 1 9 , 6-VIII-65 ; 1 ơ et 1 \%, 9-IX-65, sur Pastinaca.

Hôtes: Nonagria neurica Hb., N. geminipuncta Hatchett (Noctuidae).

Distribution : Régions chaudes d'Europe, jusqu'en Angleterre du Sud et en Allemagne du Nord; Algérie.

26) Huebneria affinis (Fall.) (Zenillia E, Exorista S, L). - 2 ?, 10-IX-64; 1 , 12-IX-64; 1 oै, 29-VIII-65, sur Pastinaca. doptères.

Hôtes: Surtout chenilles d'Arctiidae, mais aussi chenilles poilues de Microlépi-

Distribution: Europe.

27) Carcelia lucorum (Meig.). - $1 \delta, 1-\mathrm{IX}-65$, le soir sur Crepis sec ; $1 \delta$, 7-IX-65, sur Pastinaca.

Hôtes: Chenilles, surtout d'Arctiidae.

Distribution: Europe jusqu'au Sud de l'Angleterre et en Finlande ; Asie centrale (Tadjikistan).

28) Erycia festinans (Meig.). - 1 ơ, 10-IX-65, sur Daucus.

Hôtes : E. festinans est un parasite caractéristique de diverses espèces de Melitaea (Nymphalidae).

Distribution: Régions chaudes d'Europe ; atteint le Sud de la Suède et les environs de Léningrad.

29) Pales pavida (Meig.) (Ctenophorocea M). - 1 \%, 3-IX-64; 1 ;, 7-IX-64 ; 1 , 9-IX-64, toutes sur Pastniaca ; 1 \%, 25-VIII-65, sur des feuilles.

Hôtes : Espèce très polyphage, élevée des chenilles de diverses espèces de Macrolépidoptères et, en Israël, de Glyphodes unionalis (Pyralidae).

Distribution: Europe, jusqu'au Nord de l'Angleterre ; Sibérie jusqu'à la région de Vladivostok; Israël.

30) Masicera silvatica (Fall.). - 1 \%, 5-IX-64; 1 , 18-VIII-65; 1 , 30-VIII65 ; 1 \%, 7-IX-65, sur Pastinaca.

HôTES : Macrothylacia rubi L. (Lasiocampidae).

Distribution : Europe.

31) Sturmia bella (Meig.). - 1 , 5-IX-64, sur Pastinaca.

Hôtes : Chenilles, notamment de Vanessa; en Israël, Vanessa cardui L. (Kugler 1966).

Distribution: Europe, jusqu'en Allemagne du Nord; non signalée d'Angleterre ; Israël.

32) Frontina laeta (Meig.). - $1 \delta$, 4-VIII-65, sur Heracleum sphondylium.

33) Isomera blondeli (R. D.) (Gonia cognata Rond. S, H). -2 \%, 2-VIII-64 ;

HôTES: Diverses espèces de Smerinthus (Sphingidae). 
Distribution : Europe jusqu'en Angleterre méridionale, sud de la Suède, Finlande, environs de Léningrad; Sibérie (Bashkirie, Yakoutie).

1 ; , 1-IX-64; 1 ; , 3-IX-64; 1 ; , 11-IX-64; 1 \%, 12-VIII-65; 1 \%, 19-VIII-65 ; 2 + , 7-IX-65, toutes sur Pastinaca.

Hôtes : Chenilles d'Arctiidae (Coscinia bifasciata Rbr., Hipocrita jacobaeae L.).

Distribution: Régions chaudes de l'Europe, atteint l'Autriche et le sud de la Suisse.

34) Isomera cinerascens (Rond.) (Gonia S, H). - 1 \%, 11-IX-65 ; 1 б, 13-IX-65, récoltés au couchant sur des Crepis secs.

HôTES : Cirphis loreyi Dup. (Noctuidae).

Distribution : Europe méridionale jusqu'en Hongrie ; capturée également à Versailles. Signalée hors d'Europe en Afrique du Nord, au Congo, à Madagascar, aux Indes, au Viet-nam et à Formose.

35) Spallanzania hebes (Fall.) (Cnephalia S, Gonia H). - 1 ઈ', 22-VIII-64 sur Pastinaca, 1 б, 20-VIII-65 sur Eryngium campestre.

Hôtes : Noctuidae, en particulier Agrotis segetum Schiff.

Distribution : Europe jusqu'en Finlande, mais absente d'Angleterre ; Afrique du Nord ; Israël, Asie centrale (Tadjikistan).

36) Erythrocera nigripes (R. D.) (Pexomyia rubifrons Perr. S.). -1 d, 30-VIII65 sur Pastinaca.

Hôtes : Inconnus.

Distribution: Europe, jusqu'en Allemagne au Nord.

\section{ECHINOMYIINI}

37) Echinomyia grossa (L.) (Tachina M). - 1 \&, 20-VIII-64, sur Pastinaca.

Hôtes : Chenilles de Lasiocampidae et Sphingidae.

Distribution : Europe jusqu'en Ecosse ; Russie, Sibérie.

38) Echinomyia fera (L.) (Tachina M). - Espèce abondante à Richelieu pendant les deux étés 1964 et 1965 ; récoltée sur Pastinaca, Daucus et Eryngium.

Hôtes: Chenilles, en particulier de Noctuidae.

Distribution: Toute la région paléarctique, y compris Israël.

39) Echinomyia magnicornis (Zett.) (Tachina vernalis R.D., M). - Abondante à Richelieu en 1964 et 1965 ; capturée sur Pastinaca, Daucus et Eryngium.

Hôtes: Chenilles de Noctuidae.

Distribution : Abondante dans l'Ouest de la région paléarctique, jusqu'au Turkes$\tan$; Afrique du Nord, Israël.

40) Echinomyia nupta Rond. (Tachina M). - 1 б, 21-VIII-64; 1 б, 24-VIII-

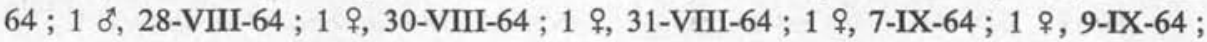
1 o, 12-VIII-65, sur Pastinaca et Daucus.

HôTES : Les hôtes de cette espèce sont difficiles à préciser car elle a longtemps été confondue avec E. magnicornis Zett. Mesnil (1966, p. 918) la distingue à nouveau et indique la différence entre les deux. 
Distribution : Sud de l'Europe, Israël.

41) Peletieria nigricornis (Meig.). -1 \&, 7-IX-64, sur Pastinaca.

Hôtes: Chenilles, en particulier des Agrotis (Noctuidae).

Distribution : Europe, y compris l'Angleterre et la Suède centrale : Asie centrale, Sibérie jusqu'en Yakoutie; Signalé d'Afrique du Nord et d'Israël.

42) Cuphocera varia argyrocephala (Macq.) (Cuphocera varia F, S, E ; Peletieria varia $\mathrm{F}, \mathrm{H}) .-1$ \%, 4-VIII-65, sur Heracleum sphondylium.

Hôtes : Chenilles de Cirphis loreyi Dup. (Noctuidae).

Distribution : Europe méridionale, Israël ; espèce abondante en Afrique tropicale, aux Indes, au Vietnam et à Formose.

43) Linnaemyia retroflexa Pand. - L'une des Tachinaires les plus répandues à Richelieu, capturée en 1964 et 1965, en particulier sur Pastinaca.

Hôtes : Probablement Noctuidae.

Distribution : Régions chaudes de l'Europe (incl. environs de Paris, Vienne...), et également environs de Léningrad, Kharkov, Kirov, Altai.

et également environs de Léningrad, Kharkov, Kirov, Altai.

44) Linnaemyia pudica (Rond.). - 1 б, 4-VIII-65; 1 ઠै, 25-VIII-65, sur Pastinaca.

Hôtes : Inconnus.

Distribution: Europe centrale, sud de l'Angleterre, Allemagne du Nord Est ; non signalée de Scandinavie.

45) Linnaemyia media Zimin. - 1 \&, 10-IX-64 ; 1 \%, 14-VIII-65 ; 1 \%, 20-VIII$65 ; 1$ o, 29-VIII-65, sur Pastinaca.

Hôtes : Inconnus.

Distribution: Décrite en 1954 d'après des spécimens récoltés à Vladivostok et dans la région de l'Amour, cette espèce se trouve aussi, selon Herting (1961) dans les régions chaudes d'Europe, telles que Vienne, Tyrol méridional, Pyrénées (Vernet-lesBains).

46) Chrysocosmius auratus (Fall.) (Chrysomopsis E, Chrysosoma L). $-1 \delta$, 18-VIII-64 ; 1 ઈో, 1-IX-65 sur Pastinaca.

Hôtes: Chenilles de Geometridae.

Distribution: Régions chaudes d'Europe ; atteint la Suède centrale.

47) Ernestia radicum (F.) (Platychira S). - 1 o, 25-VIII-65, sur Pastinaca.

Hôtes: Chenilles des Arctiidae.

Distribution: Régions tempérées d'Europe; atteint l'Ecosse, la Suède centrale et la Finlande; Caucase, Sibérie (Tonsk, région du lac Baïkal).

48) Ernestia consobrina (Meig.) (Platychira S), - 1 \&, 18-VIII-65 ; 1 o , 21-VIII$65 ; 1$ \&, 28-VIII-65; 1 o, 9-IX-65, sur Pastinaca.

Hôtes : E. consobrina est un parasite spécifique de Mamestra (Noctuidae).

Distribution : Europe centrale; atteint le Sud de l'Angleterre, la Suède centrale et la Finlande ; Kazakhstan; Vladivostok.

49) Nemoraea pellucida (Meig.). - 1 \&, 24-VIII-64; 1 శ, 10-IX-64 sur Pastinaca. 
Hôtes : Chenilles de Noctuidae, Lymantriidae, Geometridae.

Distribution: Europe jusqu'en Angleterre et Suède centrale.

50) Zophomyia temula (Scop.). - 1 o', 9-VIII-65 sur Pastinaca.

HôTes : Inconnus.

Distribution : Europe, y compris Ecosse, Suède centrale et Finlande, Kazakhstan ; Sibérie (Altaï, Toungouska).

51) Macquartia tenebricosa (Meig.) (nitida Zett. S). -2 \&, 12-IX-64 au couchant sur Crepis sec.

Hôtes: Larves de Chrysomela (Chrysomelidae).

Distribution : Europe jusqu'en Ecosse et dans le Nord de la Scandinavie ; Israël ; Kazakhstan septentrional; Sibérie occidentale.

52) Macquartia chalconota (Meig.). - 1 \%, 11-IX-64; 1 , 9-IX-65 sur Pastinaca.

HôTES : Inconnus.

Distribution : Espèce abondante en Europe, atteint le nord-ouest de l'Allemagne et le sud de la Suède; non signalée d'Angleterre.

53) Macquartia grisea (Fall.). - 1 o , 6-IX-65 sur Pastinaca.

Hôtes : Larves de Chrysomela (Chrysomelidae).

Distribution : Europe, y compris la Suède centrale.

54) Loewia phaeoptera (Meig.). - 1 \&, 8-IX-64 ; 1 ઠै, 12-IX-65 sur Pastinaca.

Hôtes: Inconnus, l'espèce voisine, L. foeda (Meig.) est parasite de Chilopodes du genre Lithobius.

Distribution: Europe jusque dans le nord de l'Angleterre, la Suède centrale et la Finlande.

55) Neaera laticornis (Meig.) (albicolis Meig. E). - 1 ઈ, 25-VIII-65 sur Pastinaca.

Hôtes : Chenilles d'Epiblema fulvana Steph. (Tortricidae).

Distribution : Espèce rare, connue du sud de l'Angleterre, de France et de Russie.

56) Solieria pacifica (Meig.) (tibialis v. Ros. S, Leskia tibialis v. Ros. E, Myobia tibialis v. Ros. L). - $1 \delta^{*}$, 9-VIII-65 ; 1 ठ, 10-IX-65 sur Pastinaca.

Hôtes : Chenilles d'Olethreutes lucivagana Z. (Tortricidae).

Distribution : Europe centrale jusqu'au Danemark et dans le sud de l'Angleterre, environ de Léningrad.

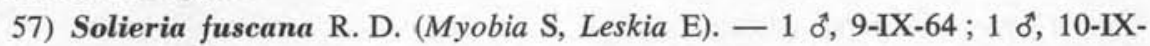
64 sur Pastinaca.

HôTES : Inconnus.

Distribution: Europe centrale, environs de Léningrad; non signalée en Scandinavie; Kazakhstan septentrional ; Sibérie occidentale.

58) Solieria vacua (Rond.) (Myobia S, Leskia $\mathrm{E})$. — Très abondante à Richelieu en 1964 et 1965 sur Pastinaca.

Hôtes : Inconnus.

Distribution: Europe, de l'Italie au sud de l'Angleterre. 
59) Bithia spreta (Meig.). - 1 ơ, 27-VIII-64 sur Pastinaca ; 1 , 3-VIII-65 sur Achillea millefolium ; 1 q, 3-VIII-65 sur Leucanthemum vulgare ; 1 d, 14-VIII-65 sur Daucus carota ; 1 ठ, 4-IX-65 le soir sur Crepis sec ; 1 \%, 9-IX-65 sur Pastinaca.

Hôtes : Inconnus.

Distribution: Europe jusqu'au sud de l'Angleterre et de la Suède ; environs de Léningrad.

\section{VORIINI}

60) Voria ruralis (Fall.). - 1 \&, 12-LX-64; 1 ఠ, 29-VIII-65; 1 ๙, 7-IX-65 ; 1 ơ, 10-IX-65 tous sur Pastinaca.

Hôtes : Chenilles, notamment de Plusia (Noctuidae); en Israël, élevée de Plusia chalcytes Esp.

Distribution : Europe ; atteint l'Ecosse et la Suède centrale ; Asie centrale (Kazakhstan) ; Israël.

61) Athricia trepida (Meig.) (Voria S, L, E). - 1 \&, 27-VIII-64 sur Pastinaca. Hôtes : Chenilles de Noctuidae.

Distribution: Europe jusqu'au sud de l'Angleterre et dans le nord de la Scandinavie; Sibérie (région de Vladivostok); Israël.

62) Wagneria nigrans (Meig.) (carbonaria Panz. S, L, E). -1 đ, 3-VIII-65, le soir sur Crepis sec.

Hôtes: Chenilles d'Agrotis (Noctuidae).

Distribution: Cette espèce a une très grande distribution; répandue en Europe, elle y atteint le sud de l'Angleterre et le nord de la Suède ; elle est connue d'Israël, de toute l'Afrique et du nord des Indes.

63) Eriothrix rufomaculatus (Deg.). - Espèce très abondante à Richelieu en 1964 et 1965 , récoltée pendant le jour sur Pastinaca et plus rarement le soir sur Crepis sec.

Hôtes : Non connus avec certitude.

Distribution: Europe y compris l'Ecosse et le sud de la Scandinavie ; Sibérie occidentale ; région du lac Baïkal.

64) Freraea albipennis (Zett.). - $14 \delta^{8} \delta^{7}$ et 6 우 du 9 au 12-IX-65, C. Dupuis leg., à terre, sous des touffes d'Ormenis mixta, plus rarement sur les fleurs elles-mêmes.

Hôres : Carabidae adultes.

Distribution : Europe, jusqu'en Ecosse, Suède centrale et aux environs de Léningrad.

\section{DEXIINI}

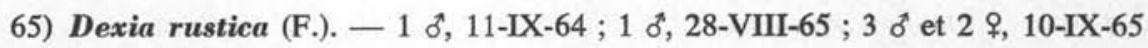
sur Daucus.

Hôtes: Larves de Scarabaeidae. 
Distribution : Europe jusqu'au sud de l'Angleterre, en Suède centrale et aux environs de Léningrad; Sibérie jusqu'à la région de Vladivostok.

66) Billea pectinata (Meig.). - 1 , , 5-VIII-65 ; 1 \%, 19-VIII-65 ; 1 \%, 29-VIII65 sur Pastinaca.

Hôtes: Larves de Scarabaeidae.

Distribution: Europe méridionale et régions chaudes de l'Europe centrale.

67) Billea triangulifera (Zett.). $-7 q$ du 1 au 10-IX-64 sur Daucus.

Hôtes: Larves de Cerambycidae.

Distribution : Europe centrale, atteint le nord de la Suède ; environs de Léningrad.

68) Dinera grisescens (Fall.). - 1 ఠ, 29-VIII-65; 1 \&, 13-IX-65 le soir sur Crepis sec.

Hôtes: Larves de Harpalus (Carabidae).

Distribution : Europe, atteint le sud de l'Angleterre, la Suède centrale et les environs de Léningrad; Sibérie (région de Vladivostok).

\section{STRONGYGASTRINA (1)}

69) Tamiclea globula (Meig.). - 1 \&, 11-VIII-63 sur Daucus (C. Dupuis leg.). HôTES: Fourmis adultes (Lasius niger L.).

Distribution: Régions chaudes d'Europe ; atteint l'Allemagne, les environs de Léningrad, Moscou et l'Ukraine.

70) Tamiclea celer (Meig.). - 1 d, $15-\mathrm{V}-64$ sur Erigeron canadensis, et 1 t, 16-V-64 sur Torilis anthriscus (C. Dupuis leg.).

Hôtes : Inconnus.

Distribution : Europe centrale, y compris la Scandinavie et les environs de Léningrad.

71) Melizoneura albipennis (R. D.). - 1 o, 5-VI-65 sur Senecio (C. Dupuis leg.).

Hôtes: Imagos de Serica (Coléoptères Scarabaeidae) (Cf. van Emden 1950).

Distribution : Europe méridionale.

\section{Résumé}

En août et septembre 1964 et 1965, à Richelieu (Indre-et-Loire), 71 espèces de Tachinaires (non comptés les Phasiinae) ont été récoltées, soit de jour sur les fleurs, soit au couchant sur les végétaux secs. De la liste de ces espèces, avec informations sur les hôtes et les distributions, on retiendra: Siphona delicatula, nouveau pour la faune française, Chaetina setigena, nouvelle pour les régions extra-méditerranéennes et un grand nombre d'espèces thermophiles.

(1) La position systématique de cette sous-tribu demeure sub judice; Mesnil (1966, p. 882) la joint aux Phasiini, mais Dupuis $(1963 \mathrm{~b})$ l'en écarte. 


\section{Index Bibliographique}

CAllot (J.), 1937. - Sur les parasites des Sauterelles à Richelieu (Indre-et-Loire). II. Ann. Parasitol. hum. \& comp., 15, 1937: 282.

Dupuis (C.), 1963 a. - Le Domaine et la Station de Richelieu (Indre-et-Loire). Station de Parasitol. exp. et comp. de Richelieu, Circ. 1, 1963: 2-13 (=Archives origin. du Centre Docum. C.N.R.S., $\mathrm{n}^{\circ}$ 387).

—, 1963 b. - Essai monographique sur les Phasiinae (Diptères Tachinaires parasites d'Hétéroptères. Mém. Mus. nat. Hist. nat., Paris, sér. A,, 26, 1963, 461 pp.

EMDEN (F. I. van-), 1950. - Dipterous parasites of Coleoptera. Entom. monthl. Mag., 86, 1950 : 182-206.

-, 1954. - Handbooks for the identification of British Insects. Diptera Cyclorrhapha: Calyptrata I. Tachinidae and Calliphoridae. London, 1954, 133 pp.

Herting (B)., 1960. - Biologie der westpaläarktischen Raupenfliegen (Dipt. Tachinidae). Monogr. angew. Ent., 16, 1960, 188 pp.

-, 1961. - Beiträge zur Kenntnis der europäischen Raupenfliegen (Dipt. Tachinidae). III-VI. Stuttgarter Beitr. Naturk., 65, 1961: 1-12.

Kugler (J.), 1961. - Orgyia dubia Tausch. and its parasites in Israel. Bull. Res. Counc. of Israel, $10 \mathrm{~B}, 1961$ : 62-72.

—, 1963. - Tachinidae of Israel. I. Israel Journ. Zool., 12, 1963 : 25-34.

-, 1966. - A list of parasites of Lepidoptera from Israel. Israel Jour. Entom., 1, 1966 : 75-88.

Lundbeck (W.), 1927. — Diptera Danica Part VII. Platypezidae, Tachinidae. Copenhagen $1927,570 \mathrm{pp}$.

MESNIL (L. P.), 1944-1966. - Larvaevorinae (Tachinidae). In Lindner (E.): Die Fliegen der paläarktischen Region. $64 \mathrm{~g}$. Stuttgart, $928 \mathrm{pp}$. (à suivre).

—, 1960. - Note préliminaire sur les Syphona Meig. (Dipt. Tachinidae) d'Europe et du bassin méditerranéen. Bull. Ann. Soc. roy. ent. Belg., 96, 1960: 187-192.

Schulov (A.), 1952. - Observations on the behaviour and the egg development of Tmethis pulchripennis asiaticus Uv. Bull. Res. Counc. of Israel, 2, 1952: 249-254.

Stackelberg (A. A.), 1962. - Liste des Diptères de la région de Léningrad VI. Diptera Calyptrata. Part I. Trudy zool. Inst. Akad. Nauk SSSR, 31, 1962: 318-388 (en russe).

STEIN (P.), 1924. - Die verbreitesten Tachinidien Mitteleuropas nach ihren Gattungen und Arten. Arch.f. Naturgesch., 90 A, 6, 1924, 271 pp.

Zrmin (L. S.), 1954. - Les espèces du genre Linnaemyia Rob.-Desv. (Diptera, Larvaevoridae) dans la faune de I'U.R.S.S. Trudy zool. Inst. Akad. Nauk SSSR, 15, 1954: 258-282 (en russe).

(Département de Zoologie, Université de Tel-Aviv). 